\title{
A secure "double-check" technique of bedside post pyloric feeding tube placement using transnasal endoscopy
}

\author{
Atsunori Hashimoto, Munehiko Oya, Mika Iwano, Chisako Fuse, Tomoko Inoue, Taihei Yamada, \\ Mariko Terashima, Takaaki Osako, Takahiro Ueda, Isamu Yamada, Atsunori Nakao and Joji Kotani* \\ Department of Emergency and Critical Care Medicine, Hyogo College of Medicine, 1-1, Mukogawa-cho, Nishinomiya, Hyogo 663-8501 Japan
}

(Received 17 March, 2012; Accepted 10 April, 2012; Published online 12 July, 2012)

\begin{abstract}
Enteral feeding has become an important means of providing nutritional support to seriously ill patients. Placement of the feeding tube through the pyloric ring and past the ligament of Treitz into the proximal jejunum is critical to reduce the risk of gastroesophageal regurgitation and microaspiration. We started utilizing transnasal endoscopy for intestinal feeding tube placement, placing enteral tubes for $\mathbf{4 0}$ patients between March 2008 and February 2009. Although we achieved a high success rate comparable to previous reports, we experienced several cases of failure, which was corrected with repeated endoscopy. Based on these experiences, we modified our method by adding a "doublecheck" transnasal endoscopy through the other nasal passage. After April 2010, we have placed the feeding tube by "doublecheck" method for all patients (more than 40 patients) who required transnasal endoscopic feeding tube placement. We have not experienced any misplacement in all these patients after $24 \mathrm{~h}$ later with $100 \%$ successful rate since the introduction of "doublecheck" procedure. We describe our experience with "doublecheck" transnasal endoscopic feeding tube placement, which we found to be a helpful adjunct, for patients in intensive care unit.
\end{abstract}

Key Words: enteral feeding, endoscope, intestinal tubing, transnasal, complication

E arly enteral feedings are known to improve outcomes in critically ill intensive care unit (ICU) patients having difficulty with volitional intake. ${ }^{(1,2)}$ Maintenance of gut integrity by enteral nutrition leads to less bacterial translocation and decreased systemic inflammatory response. ${ }^{(3,4)}$ Thus, enteral feeding has become an important means of providing nutritional support to seriously ill patients.

Placement of the feeding tube through the pyloric ring and past the ligament of Treitz into the proximal jejunum is critical to reduce the risk of gastroesophageal regurgitation and microaspiration, which may improve tolerance of enteral nutrition. ${ }^{(5-7)}$ Conventionally, simple insertion of a feeding tube in the stomach was performed expecting spontaneous transpyrolic migration of the tube, associated with a low success rate of approximately $5-15 \%{ }^{(8)}$ Fluoroscopic positioning has also been used for enteral feeding tube placement; however, positioning frequently fails because of functional gastric outlet obstruction, gastric dysmotility, gastric distention, and distortion of the duodenal loop. ${ }^{(9)}$ Although this fluoroscopy imaging procedure is universally performed, it requires transportation of severely ill patients to the radiology suite and nursing and respiratory support, possibly increasing risk of complications during transport.

Endoscopic placement of feeding tubes was successfully used after other methods, such as fluoroscopy, failed. More recently, a transnasal endoscopic method using a thin endoscope was developed, and reports demonstrate that the procedure duration is significantly shorter without radiation exposure and the need for transport to radiology. ${ }^{(10-12)}$ As previous reports have shown, an $84-93 \%$ correct tube positioning rate can be achieved by transnasal endoscopy. ${ }^{(9,13,14)}$

In our department, we started utilizing transnasal endoscopy for intestinal feeding tube placement, placing enteral tubes for 40 patients between March 2008 and February 2009. Although we achieved a high success rate comparable to previous reports, we experienced several cases of failure, which was corrected with repeated endoscopy. Based on these experiences, we modified our method by adding a "double-check" transnasal endoscopy through the other nasal passage, which we used on 14 patients from March 2009 to March 2010. Our novel "double-check" method enabled instant replacement in cases of incorrect positioning, avoided repeated abdominal plain X-ray, and allowed us to achieve a $100 \%$ success. In this report, we describe our experience with "doublecheck" transnasal endoscopic feeding tube placement, which we found to be a helpful adjunct, in ICU patients. We also compared the "double-check" method with a conventional method, in terms of time of procedure and successful rate (no requirement of replacement $24 \mathrm{~h}$ later).

\section{Methods}

Patients. Forty eight consecutive critically ill ICU patients at our hospital who were placed with bowel feeding tubes using nasal endoscopes between March 2009 and March 2010 were included in our review. Six cases were excluded where time was spent investigating certain complications, such as suspected bleeding or difficulty of placement. All patients received adequate cardiopulmonary monitoring including the blood pressure, oxygen saturation, and pulse rate during endoscopic procedures. We performed the endoscopic feeding tube insertion only when the patients' conditions met the following criteria; systolic blood pressure $<150 \mathrm{mmHg}$ and $>90 \mathrm{mmHg} ; \mathrm{O}_{2}$ saturation $>96 \%$; Heart rate $<120 \mathrm{bpm}$ and $>50 \mathrm{bpm}$. Patients were divided into two groups: the cases in the initial period without re-endoscopy ( 28 cases) and the latter cases with "double-check" re-endoscopy (14 cases). Informed consent was obtained from patients or their family members.

Procedure. All endoscopies were performed at the bedside

*To whom correspondence should be addressed.

E-mail: kotanijo@attglobal.net 


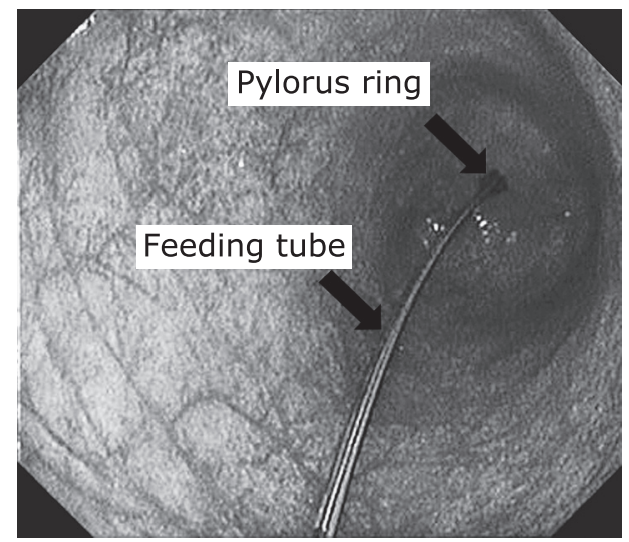

Fig. 1. Representative endoscopical image at "double check". The feeding tube was passed over the pylorus ring.

in the ICU by one or two physicians. No sedation was needed for alert patients. Topical lidocaine was sprayed into the nose and retropharynx in conscious patients. The tip of the endoscope (XP260N, Olympus Corporation, Tokyo, Japan; outer diameter $5.9 \mathrm{~mm}$ ) was passed under direct vision into one of the nasal foramen. After panendoscopic examination and cannulation of the duodenum, the tip of the endoscope was inserted into the descending part or horizontal part of duodenum, and the guidewire (Create Medic, FS-20T, 052"-3500T, Tokyo, Japan) for ileus tube (Argyle, New enteral feeding tube) was inserted through the endoscope channel as far as possible under direct observation. Then, the endoscope was removed, leaving the guidewire; the feeding tube was advanced along the guidewire, and the guidewire was then pulled out. ${ }^{(10,14,15)}$ For "double-check" re-endoscopy following placement of the feeding tube, the same endoscopy was inserted again through the other nasal foramen, and the correct tube position was verified by visualizing the tube passing the pyloric ring (Fig. 1).

Assessment. $100 \mathrm{ml}$ of contrast medium (Gatsrographin ${ }^{\circledR}$ ) was injected into the tube immediately after placement and an abdominal X-ray was performed $24 \mathrm{~h}$ later to estimate the location of the tube tip and assess regurgitation of the contrast medium into the stomach and intestinal motility. Successful placement of the nasoenteral feeding tube tip was defined as insertion into the second part of the deodenum or further down and passage of the contrast agents to the ileum, confirmed by plain abdominal $\mathrm{X}$-ray $24 \mathrm{~h}$ later. We retrospectively collected patient data such as age, gender, and background disease. The duration of each procedure was recorded.

\section{Results}

Demographic data for our patients are shown in Table 1. No significant differences between any of the variables were seen. The success rate of the patient group without "double-check" was $82.1 \%(23 / 28)$, with the tip of the catheter placed into the distal duodenum in 7 patients $(25 \%)$ and into the jejunum in 21 patients $(28 \%)$. In 5 cases $(14 \%)$, the tip of the feeding tube was misplaced in the stomach and bulbus of the duodenum. Plain X-ray taken $24 \mathrm{~h}$ later revealed remaining contrast agents in the stomach. The unsuccessful placements in these patients were corrected endoscopically the following day. The mean procedure time required was $12.4 \pm 2.6 \mathrm{~min}$ (ranging seven to $30 \mathrm{~min}$ ).

The second group of patients receiving the "double-check" technique consisted of 9 males and 5 females. Successful placement of the feeding tube was achieved in $100 \%$ patients, as verified by plain abdominal X-ray examination $24 \mathrm{~h}$ later. In one
Table 1. Patients summary

\begin{tabular}{lcc}
\hline & single-check & double-check \\
\hline patient number & 28 & 14 \\
mean age & $56.5(15-90)$ & $66.1(16-88)$ \\
male/female ratio & $21 / 7$ & $9 / 5$ \\
procedure time in minutes & $12.4 \pm 2.6$ & $13.1 \pm 2.3$ \\
admission diagnosis & 2 & \\
$\quad$ cardiac & 5 & 1 \\
respiratory & 4 & 4 \\
neurologic & 5 & 3 \\
surgical & 12 & 3 \\
$\quad$ others & 5 & 0 \\
required replacement $24 \mathrm{~h}$ later & & 3 \\
\hline
\end{tabular}

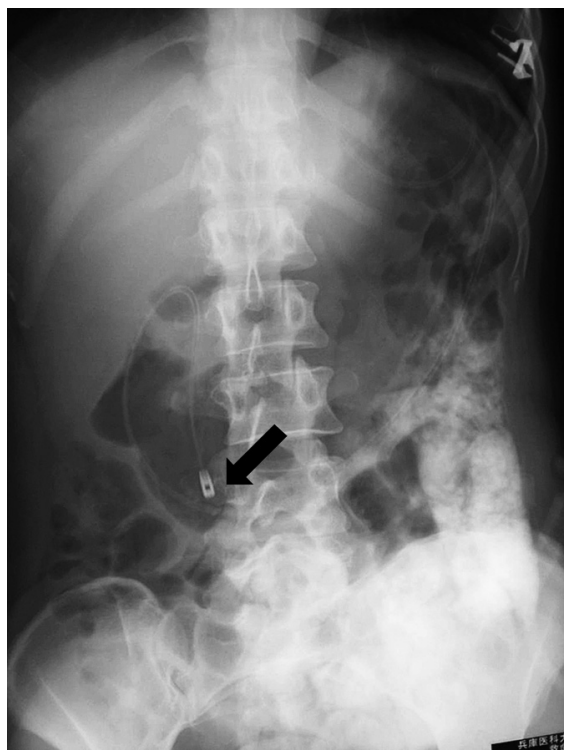

Fig. 2. Representative image of an abdominal plain X-ray performed $24 \mathrm{~h}$ later. We confirm the tip of the feeding tube located in the proximal jejunum regurgitation of the contrast medium into the stomach. Arrow indicates the tip of the feeding tube located in proximal jejunum.

case, "double-check" endoscopy revealed an incorrect position of the feeding tube, and replacement was undertaken immediately. The average procedure time (including the first placement and replacement of endoscope) was $13.1 \pm 2.3 \mathrm{~min}$ (ranging 10 to $17 \mathrm{~min}$ ). In all patients, contrast agents passed to the ileum $24 \mathrm{~h}$ later, as verified by portable plain X-ray (Fig. 2).

After April 2010, we have placed the feeding tube by "doublecheck" method for all patients (more than 40 patients) who required transnasal endoscopic feeding tube placement. We have not experienced any misplacement in all these patients after $24 \mathrm{~h}$ later with $100 \%$ successful rate since the introduction of "doublecheck" procedure. No additional medication for the treatment of complications was required in any case.

\section{Discussion}

This report demonstrates our experiences with transnasal enteral feeding tube placement and verification/correction using "doublecheck" endoscopy. This method is accurate and secure with feasibility in the ICU. Transnasal endoscopic placement of feeding 
tubes has been applied widely and been found to be effective, as it can be performed at the bedside without the need to transport or reposition the patient. At this moment, we routinely perform "double-check" endoscopy after initial placement. No fluoroscopy is used to identify the exact position of the guidewire or feeding tubes.

Endoscopic placement of feeding tubes has the additional advantage of providing valuable information on the upper gastrointestinal tract, as ICU patients frequently have upper gastrointestinal-tract pathology. Also, endoscopic placement enables insertion of the tubes to the efferent loop after gastrectomy.

Our double-check endoscopy method requires a few minutes of extra time. However, we believe that availability of rapid tube reinsertion after inadvertent displacement or replacement can compensate for this delay. Wiegand et al. ${ }^{(16)}$ first reported that endoscope position control for enteral feeding tubes with transnasal re-endoscopy has the potential to substantially save costs. Of note, the second removal of the endoscope must be conducted with maximal attention, as we experienced looping of the feeding tubes or misplacement in the stomach at the first insertion.

Recent advances in endoscopic technology have led to the production of very thin endoscopes with instrument channels, which enable exiting the intestinal tube through the instrument

\section{References}

1 Klein S, Kinney J, Jeejeebhoy K, et al. Nutrition support in clinical practice: review of published data and recommendations for future research directions. Clin Nutr 1997; 16: 193-218.

2 McClave SA, Martindale RG, Vanek VW, et al. Guidelines for the provision and assessment of nutrition support therapy in the adult critically ill patient: society of critical care medicine (SCCM) and American society for parenteral and enteral nutrition (A.S.P.E.N.). JPEN Parenter Enteral Nutr 2009; 33: 277-316.

3 Moore FA, Feliciano DV, Andrassy RJ, et al. Early enteral feeding, compared with parenteral, reduces postoperative septic complications. The results of a meta-analysis. Ann Surg 1992; 216: 172-183.

4 Windsor AC, Kanwar S, Li AG, et al. Compared with parenteral nutrition, enteral feeding attenuates the acute phase response and improves disease severity in acute pancreatitis. Gut 1998; 42: 431-435.

5 Davies AR, Froomes PR, French CJ, et al. Randomized comparison of nasojejunal and nasogastric feeding in critically ill patients. Crit Care Med 2002; 30: 586-590.

6 Heyland DK, Drover JW, MacDonald S, et al. Effect of postpyloric feeding on gastroesophageal regurgitation and pulmonary microaspiration: results of a randomized controlled trial. Crit Care Med 2001; 29: 1495-1501.

7 Hsu CW, Sun SF, Lin SL, et al. Duodenal versus gastric feeding in medical intensive care unit patients: a prospective, randomized, clinical study. Crit Care Med 2009; 37: 1866-1872.

8 DiSario JA, Baskin WN, Brown RD, et al. Endoscopic approaches to enteral nutritional support. Gastrointest Endosc 2002; 55: 901-908.

9 O'Keefe SJ, Foody W, Gill S. Transnasal endoscopic placement of feeding channel. ${ }^{(12)}$ These new endoscopes can potentially allow advancement of the guidewire and the nasoenteral feeding tube deeper into the duodenum and jejunum.

Contraindications (exclusion criteria) are severe coagulopathy, history of nasopharyngeal trauma or surgery, and the potential need for a therapeutic endoscopic procedure. Reported complications of this new technique may be similar to those of conventional transnasal endoscopic feeding, including nasopharyngeal erosion, aspiration, diarrhea, metabolic derangement, and epistaxis. ${ }^{(17,18)}$ We acknowledge that our study has been too small and underpowered to conclude that the novel "double check method" was secure and safe. However, as far as our experience was concerned, both procedures were associated with minimal complications and stable vital signs, although the background disease of the patients varied in this study.

In conclusion, our double-check method has major advantages over and is more secure and reliable than the current method of endoscopic placement.

\section{Conflict of Interest}

No potential conflicts of interest were disclosed.

tubes in the intensive care unit. JPEN J Parenter Enteral Nutr 2003; 27: 349 354.

10 Stark SP, Sharpe JN, Larson GM. Endoscopically placed nasoenteral feeding tubes. Indications and techniques. Am Surg 1991; 57: 203-205.

11 Montecalvo MA, Steger KA, Farber HW, et al. Nutritional outcome and pneumonia in critical care patients randomized to gastric versus jejunal tube feedings. The critical care research team. Crit Care Med 1992; 20: 1377-1387.

12 Zick G, Frerichs A, Ahrens M, et al. A new technique for bedside placement of enteral feeding tubes: a prospective cohort study. Crit Care 2011; 15: R8.

13 Fang JC, Hilden K, Holubkov R, DiSario JA. Transnasal endoscopy vs. fluoroscopy for the placement of nasoenteric feeding tubes in critically ill patients. Gastrointest Endosc 2005; 62: 661-666.

14 Ott DJ, Mattox HE, Gelfand DW, Chen MY, Wu WC. Enteral feeding tubes: placement by using fluoroscopy and endoscopy. AJR Am J Roentgenol 1991; 157: 769-771.

15 Külling D, Bauerfeind P, Fried M. Transnasal versus transoral endoscopy for the placement of nasoenteral feeding tubes in critically ill patients. Gastrointest Endosc 2000; 52: 506-510.

16 Wiegand N, Bauerfeind P, Delco F, Fried M, Wildi SM. Endoscopic position control of nasoenteral feeding tubes by transnasal re-endoscopy: a prospective study in intensive care patients. Am J Gastroenterol 2009; 104: 1271-1276.

17 DiSario JA, Baskin WN, Brown RD, et al. Endoscopic approaches to enteral nutritional support. Gastrointest Endosc 2002; 55: 901-908.

18 Levy H. Nasogastric and nasoenteric feeding tubes. Gastrointest Endosc Clin N Am 1998; 8: 529-549. 